\title{
Development of Simple Optical Sensor for Measurement of Sugar Content Using Bent Optical Fiber, Light Emitting Diode and Photo Diode
}

\author{
Ken-ichiro SUEHARA*,†, Takaharu KAMEOKA** and Atsushi HASHIMOTO* \\ * Department of Life Sciences, Graduate School of Bioresources, Mie University, Japan \\ ** Department of Environmental Science and Technology, Graduate School of Bioresources, Mie University, Japan
}

Received February 28, 2017, Accepted November 28, 2017

\begin{abstract}
To construct a simple optical sensor for measurement of the sugar content in an aqueous solution, a bent optical fiber was used as the sensor. The attenuation ratio, i.e., the differences in the light intensity through the bent optical fiber when the sensitive part (bent part) was soaked in a reference material and sample solution, was closely related of the solute content in the samples. However, slightly difference in the light intensity were observed in the practical application because the sugar content in foods and other bio-systems was much lower than that in the tested samples. Therefore, we constructed a sensor system using a light emitting diode (LED), photo diode (PD) and simple electronic circuit as a differential amplifier. As a result, measurement of the sugar content ranging from 0 to $30 \mathrm{~g} / \mathrm{dm}^{3}$ was successful and excellent agreement between the FT-IR method as the conventional method and the sensor method using an operational amplifier (OP-amp) was observed. In addition, the time courses of the sugar content and the uptake rate of plant cells were able to be calculated during the cultivation process. The process monitoring for the fermentation process management will be possible by using this sensor. These results suggested that the developed sensor using a bent optical fiber is applicable as a simple and disposable optical sensor.
\end{abstract}

KEYWORDS: bent optical fiber, optical sensing, optical sensor, LED, sugar content

\section{Introduction}

Optical sensing, such as a spectroscopic analysis and colorimetric method, is widely used in various fields, for example, management of agricultural and food pro-

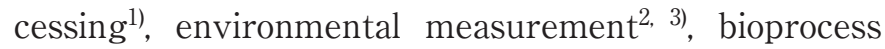
measurement and control ${ }^{4)}$, medical monitoring ${ }^{5,6)}$, etc. The optical sensing is advantageous in that a simple, rapid, chemical free, non-invasive, and non-destructive measurement is possible. In addition, the obtained data have a high affinity with Information Communication Technology (ICT) because the optical sensing data are recorded as digital information. In the application of new digital technologies, such as the concept of Internet of Things (IoT), vast amounts of various types of digital data are produced and the aggregate of the data is called Big Data ${ }^{7}$. The optical sensing method will be one of the important measurement tools for the IoT concept with the Big Data application in the field of agricultural and food processing ${ }^{8)}$.

The optical fiber is an essential intelligent technology for optical communications in the ICT field. The underground optical fiber is useful as a sensor for the detection of ground strain ${ }^{9)}$ and displacement of ground positions ${ }^{10)}$. The optical fiber, which was specifically processed, is used to measure physical and chemical properties such as refractive index ${ }^{11)}$, temperature ${ }^{12)}$ and humidity ${ }^{13)}$. The clad of the optical fiber was removed and the sensing materials, such as a fluorescence enhancer for specific materials and polymer film containing an organic pigment for humidity detection, were coated on the naked fiber core ${ }^{11}$. In the case of the refractive index sensor, gold (or platinum) was coated on the fiber core. For the other cases, the fiber shape was precisely processed ${ }^{10,13)}$. In these sensor systems, complicated signal processing is generally required for the detection of the target substrates or the properties. In our previous study, an air-oil and oil-water interface detector composed of a plastic optical fiber, light emitting diode (LED), photo diode (PD) and five analog electric circuits, was developed and the sensor was applied to measurement of the oil layer thickness of kitchen wastewater ${ }^{14)}$. The plastic optical fiber, which is not processed, was

\footnotetext{
${ }^{\dagger}$ Corresponding Author: Ken-ichiro Suehara suehara@bio.mie-u.ac.jp
} 
used to create the sensitive part of the sensor except that the fiber was bent. In other words, forming of the sensitive part is very simplified and the sensitive part of the sensor can be more easily supplied at a low price and in large quantities. In addition, signal processing for the sensor was able to be considerably simplified because the air-oil and oil-water interfaces are able to be detected by the light intensity changing through the bent optical fiber ${ }^{14)}$. If the measurement of the consecutive content of a solute in the solution is possible using the developed sensor in our previous study ${ }^{14}$, a simple and disposable optical sensor will be applicable for foods and other bio-systems including environmental measurement in the IoT concept.

The light emitting diode (LED) is widely used in the field of applied optical radiation such as general lighting, flat-panel displays, light source for evaluation of color appearance of objects ${ }^{15}$, indicator and traffic light ${ }^{16)}$. The LED is also used in a plant factory which requires precision control of the light environment ${ }^{17}$. The application of LEDs as a stable light source is very useful because LEDs may have a very long life expectancy and the light-emitting intensity is able to be controlled only by amperage regulation of the current supplied to the LED. Therefore, the combination of the LED as the light source for the optical sensor and PD as the detector has recently attracted interest to construct various optical sensors ${ }^{18-20)}$.

The propose of this study is to develop a simple optical sensor using an LED, PD and bent optical fiber to measure the content of a bio-related substance in an aqueous solution which is considered to be in foods and other bio-reaction systems. In particular, sugar is the most essential bio-related substance for living organisms including agricultural products, cells, and the other living things because sugar is the main material for the starting point of the metabolism reaction. During the fermentation (cell cultivation) process, sugar is mainly used as the carbon source for cell growth, and maintains and allows the metabolic process in cells to continue. In general, sugar content in the fermented culture broth is measured by enzymatic ${ }^{21)}$ or by electrochemical detection ${ }^{22}$. These methods have a high detection sensitivity (micro molar order). However, it is one of the serious problems for the continuous monitoring that the activity is not stable in foods and the other biological fluids. In addition, the sensor should be sterilized to avoid the microbial contamination. Although the non-enzymatic method was developed ${ }^{23-25)}$ in resent study, stability of the sensitive materials is also serious problem because these methods are detected based on the chemical or physical interaction between sugar and the sensitive material.

Optical sensing is one of the suitable techniques for measuring the content of a bio-related substance such as the sugar during the bioprocess progression because a simple, rapid, and non-destructive measurement is possible. In addition, the target substance might be able to be detected by an optical sensor without using the sensing materials. Therefore, we tried to develop an optical sensor to measure the sugar content in an aqueous solution base with the interface detector which we developed in a previous study ${ }^{14)}$. A fundamental performance test of the bent optical fiber used as the sensor was first carried out using an aqueous solution of sucrose, sodium chloride and mixture of sucrose and sodium chloride. A prototype sensor system was then constructed using an LED, PD and simple electronic circuit (differential amplifier) using an operational amplifier (OP-amp) and was applied to the monitoring of the sugar content in a plant cell culture broth which is one of the typical multi-component samples.

\section{Materials and methods 2.1 Materials}

Aqueous solutions of sucrose, sodium chloride $(\mathrm{NaCl})$ and a mixture of sucrose and $\mathrm{NaCl}$ were used as the samples. Special grade sucrose and $\mathrm{NaCl}$ were purchased from Wako Pure Chemical Industries, Ltd. (Osaka, Japan). The contents of the sucrose and $\mathrm{NaCl}$ of the prepared aqueous solutions as the samples are summarized in Table 1. Sugar and salt are the main components in the bio-materials such as food and culture media. In addition, the interaction between the sugar and salt in the sugar-salt aqueous solution might be observed in the optical sensing data ${ }^{26)}$. Therefore, the mixing ratio was changed dynamically as shown in Table 1 for the fundamental performance test of the optical sensor using bent optical fiber.

Arabidopsis thaliana MM2d was used as the plant cell cultivation $^{27}$. The cells were sub-cultured in the $\mathrm{Mu}$ rashige and Skoog (MS) basal salts medium ${ }^{28)}$ containing $30 \mathrm{~g} / \mathrm{dm}^{3}$ sucrose, $0.5 \mathrm{mg} / \mathrm{dm}^{3}$ a-naphthalene acetic acid and $0.05 \mathrm{mg} / \mathrm{dm}^{3}$ kinetin. For the sub-cultivation, $4.0 \mathrm{~cm}^{3}$ of the 7-day-old inoculums in the MS medium was inoculated in $100 \mathrm{~cm}^{3}$ of separate fresh mediums in $300 \mathrm{~cm}^{3}$ flasks, and the pre-cultivation was carried out at $300 \mathrm{~K}$ using a rotary shaker (130 rpm) under dark conditions. For the cultivation, cells sub-cultured for 7 days were washed using the MS medium without containing sugar, and $1.6 \mathrm{~cm}^{3}$ of the suspension was inoculated in $40 \mathrm{~cm}^{3}$ of separate fresh medium in $100 \mathrm{~cm}^{3}$ Erlenmeyer flasks. The cells were grown in the dark under agitation at $130 \mathrm{rpm}$ and $300 \mathrm{~K}$. To get the various states of the culture broth, the broth in the flask was diachronically sampled.

\subsection{Experimental apparatus}

A plastic optical fiber (SK60, Mitsubishi Rayon Co., Ltd., Tokyo) was used as the sensor, and the optical 
Table 1 Constituents of sugar-salt aqueous solutions.

\begin{tabular}{|c|c|c|c|c|c|c|c|c|}
\hline & \multicolumn{2}{|c|}{ Sucrose content } & \multicolumn{2}{|c|}{$\mathrm{NaCl}$ content } & \multirow{2}{*}{$\begin{array}{l}\text { Density } \\
{\left[\mathrm{g} / \mathrm{cm}^{3}\right]}\end{array}$} & \multirow{2}{*}{$\begin{array}{c}\text { Brix\% } \\
{[\%]}\end{array}$} & \multirow{2}{*}{$\begin{array}{c}\mathrm{EC} \\
{[\mathrm{S} / \mathrm{m}]}\end{array}$} & \multirow{2}{*}{$\begin{array}{c}\text { Factor } \\
{[-]}\end{array}$} \\
\hline & {$\left[\mathrm{mol} / \mathrm{dm}^{3}\right]$} & [wt\%] & {$\left[\mathrm{mol} / \mathrm{dm}^{3}\right]$} & [wt\%] & & & & \\
\hline Water & 0.000 & 0.00 & 0.00 & 0.00 & & & & 1.000 \\
\hline \multirow[t]{4}{*}{ Sucrose } & 0.125 & 4.20 & 0.00 & 0.00 & 1.01 & 4.20 & & 0.9689 \\
\hline & 0.250 & 8.30 & 0.00 & 0.00 & 1.03 & 8.30 & & 0.9425 \\
\hline & 0.375 & 12.3 & 0.00 & 0.00 & 1.04 & 12.3 & & 0.9159 \\
\hline & 0.500 & 16.1 & 0.00 & 0.00 & 1.06 & 16.1 & & 0.8901 \\
\hline \multirow[t]{4}{*}{$\mathrm{NaCl}$} & 0.000 & 0.00 & 0.50 & 2.90 & 1.02 & 3.60 & 4.39 & 0.9861 \\
\hline & 0.000 & 0.00 & 1.00 & 5.60 & 1.04 & 6.70 & 7.70 & 0.9774 \\
\hline & 0.000 & 0.00 & 2.00 & 10.9 & 1.07 & 12.7 & 13.70 & 0.9562 \\
\hline & 0.000 & 0.00 & 4.00 & 20.4 & 1.15 & 23.1 & 19.51 & 0.9134 \\
\hline \multirow[t]{16}{*}{ Sucrose $+\mathrm{NaCl}$} & 0.125 & 4.10 & 0.50 & 2.90 & 1.03 & 7.70 & 3.98 & 0.9604 \\
\hline & 0.125 & 4.10 & 1.00 & 5.60 & 1.05 & 10.7 & 7.10 & 0.9512 \\
\hline & 0.125 & 3.90 & 2.00 & 10.7 & 1.09 & 16.4 & 11.96 & 0.9312 \\
\hline & 0.125 & 3.70 & 4.00 & 20.1 & 1.16 & 26.5 & 17.90 & 0.8865 \\
\hline & 0.250 & 8.20 & 0.50 & 2.80 & 1.05 & 11.5 & 3.75 & 0.9327 \\
\hline & 0.250 & 8.00 & 1.00 & 5.50 & 1.07 & 14.5 & 6.74 & 0.9238 \\
\hline & 0.250 & 7.70 & 2.00 & 10.6 & 1.11 & 20.0 & 11.34 & 0.9038 \\
\hline & 0.250 & 7.30 & 4.00 & 19.8 & 1.18 & 29.8 & 16.44 & 0.8590 \\
\hline & 0.375 & 12.1 & 0.50 & 2.80 & 1.06 & 15.4 & 3.43 & 0.9065 \\
\hline & 0.375 & 11.8 & 1.00 & 5.40 & 1.08 & 18.3 & 6.10 & 0.8968 \\
\hline & 0.375 & 11.5 & 2.00 & 10.4 & 1.12 & 23.6 & 10.2 & 0.8759 \\
\hline & 0.375 & 10.8 & 4.00 & 19.6 & 1.19 & 33.0 & 14.8 & 0.8314 \\
\hline & 0.500 & 4.10 & 0.50 & 2.90 & 1.05 & 19.2 & 3.13 & 0.8806 \\
\hline & 0.500 & 4.10 & 1.00 & 5.60 & 1.10 & 21.9 & 5.52 & 0.8708 \\
\hline & 0.500 & 3.90 & 2.00 & 10.7 & 1.14 & 27.0 & 9.29 & 0.8492 \\
\hline & 0.500 & 3.70 & 4.00 & 20.1 & 1.21 & 36.1 & 13.3 & 0.8057 \\
\hline
\end{tabular}
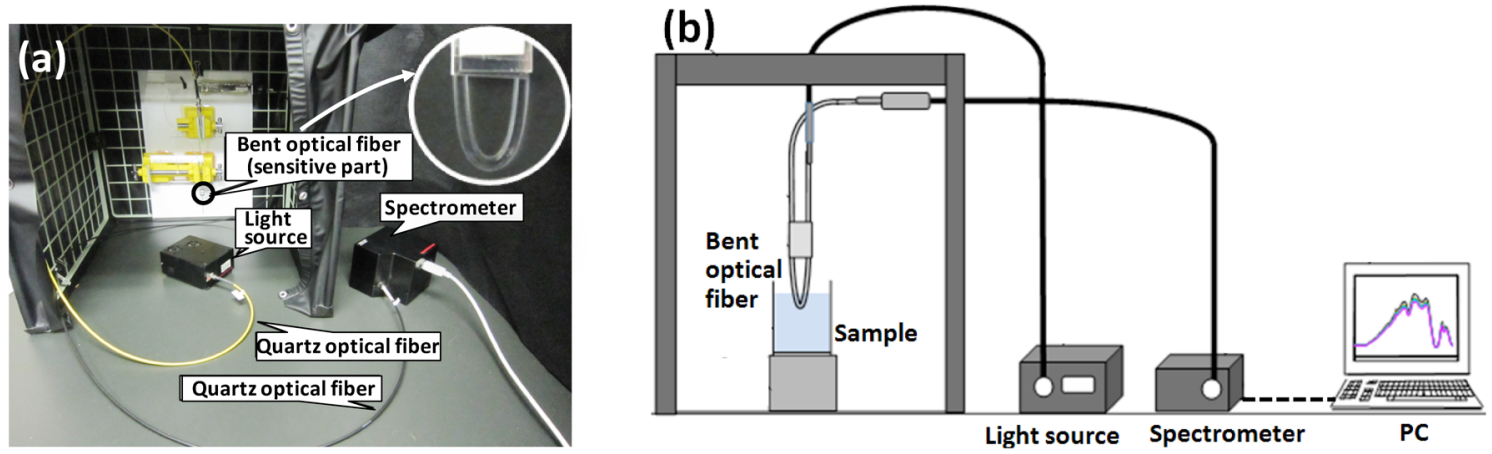

Figure 1 Overview and schematic diagram of experimental apparatus.

fiber was bent for creating the sensitive part. To check the fundamental performance of the sensor, the experimental apparatus was constructed as shown in Figure 1. Both ends of the bent optical fiber were connected to a light source (L10671, Hamamatsu Photonics K. K., Hamamatsu, Japan) and spectrometer equipped with a CCD image sensor with a spectral response from 320 to $1000 \mathrm{~nm}$ (C10083CAH, Hamamatsu Photonics K. K., Hamamatsu, Japan) through the quartz optical fiber. The output signal from the spectrometer was pro- cessed using attached software and the light intensity spectrum was recorded by the $\mathrm{AD}$ count value scaled in 16 digital bits.

\subsection{Conventional analysis of sugar concentration in culture broth}

The sugar contents of the sampled culture broth were determined by the FT-IR/ATR (Attenuated Total Reflection) method as previously described ${ }^{29)}$. For the analysis, an FT-IR spectrometer (Nicolet Magna 750, 
Thermo Fisher Scientific Inc., USA) was equipped with a $\mathrm{KBr}$ beam splitter, and a deuterated triglycine sulfate $\mathrm{KBr}$ detector was used to obtain the spectra. The ATR spectra were obtained using a horizontal zinc selenide ATR sampling accessory (SPECACLAMP ATR 11080, SPECAC Ltd., UK). The dry cell weight of the culture broth was gravimetrically measured after collection by centrifugation, washed and the excess water removed.

The logistic function expressed by Eq. (1) was applied to the plots of the dry cell weight and sugar uptake versus the cultivation time described in our previous report ${ }^{30)}$

$$
W=\frac{W_{i n i}-W_{f i n}}{1+e^{\left(\left(t-t_{0}\right) / w\right)}}+W_{f i n}
$$

where $W\left[\mathrm{~g} / \mathrm{dm}^{3}\right]$ is the dry cell weight or the total sugar content. $t[\mathrm{~d}]$ is the cultivation time. The parameters $t_{0}$ and $w$, respectively, denote the inflection point of the time course and the time intervals that define its curve width. Moreover the subscripts ini and fin indicate the value at the initial and final stages, respectively. The growth and sugar uptake rates of the cells were calculated by differentiating Eq. (1) with the cultivation time, $t$.
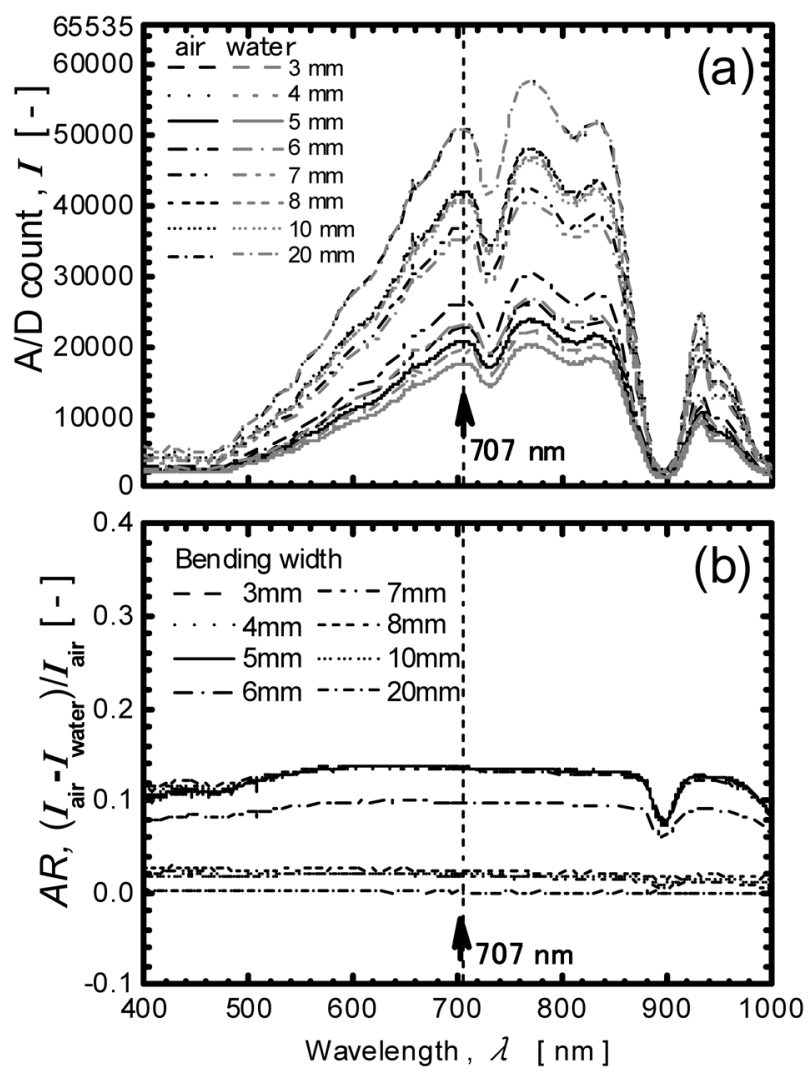

Figure 2 Spectra of light intensity passing through the bent optical fiber (a) and calculated attenuation ratio, $A R$, of the light intensity (b).

\section{Experimental results}

3.1 Determination of optimal bending width of the plastic optical fiber

To create the sensitive part of the sensor, the plastic optical fiber was gradually bent. Figure 2(a) shows the light intensity spectra passing through the bent plastic optical fiber which is the sensitive part that was soaked in air and water. The vertical axis of Figure 2(a) is the $\mathrm{AD}$ count values obtained from the spectrometer which recorded the light intensity scaled in 16 digital bits. The spectral pattern is almost the same as the light source except at around $900 \mathrm{~nm}$. The absorption at around $900 \mathrm{~nm}$ might be caused by the plastic as the fiber material.

The differences in the light intensity when the sensitive part was soaked in air and water are also displayed in Figure 2(b). In the figure, the attenuation ratio, $A R$, of the light intensity in the case of air and water was defined by the following equation

$$
A R=\frac{I_{r}-I_{s}}{I_{r}}
$$

where $I_{\mathrm{r}}$ and $I_{\mathrm{s}}$ are the light intensity through the bent plastic optical fiber when the sensitive part was soaked in the reference (air) and sample (water) materials, respectively. To determine the optimal bending width of the sensitive part, the relationship between the bending width and the attenuation ratio at $707 \mathrm{~nm}$ was plotted (Figure 3). As a result, it was determined that the plastic optical fiber with bent a width of $5 \mathrm{~mm}$ maximized the $A R$ value.

\subsection{Fundamental performance test using sugar-salt aqueous solutions}

Figure 4 shows the relationship between the $A R$ values and the mass percentage, density or Brix \% of the sucrose aqueous solutions. The $A R$ value is closely related to the sucrose content in the aqueous solution.

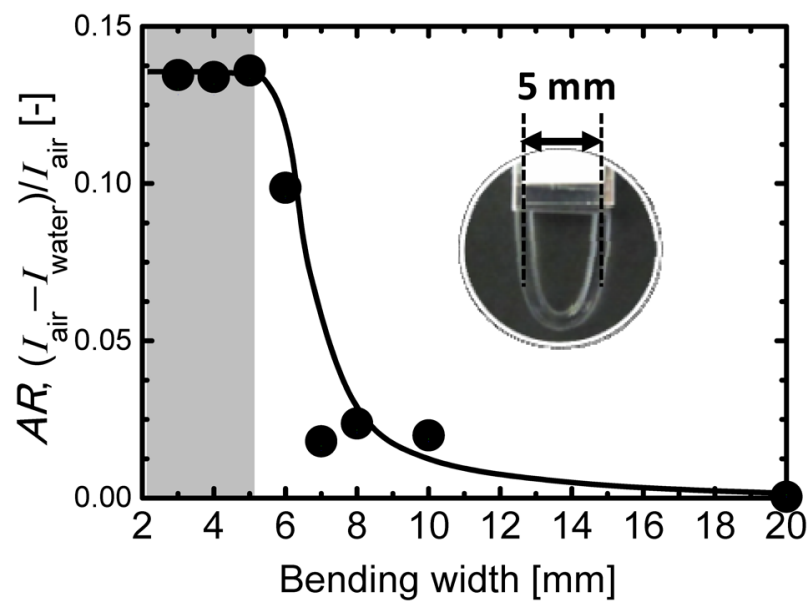

Figure 3 Relationship between bending width of optical fiber and $A R$. 


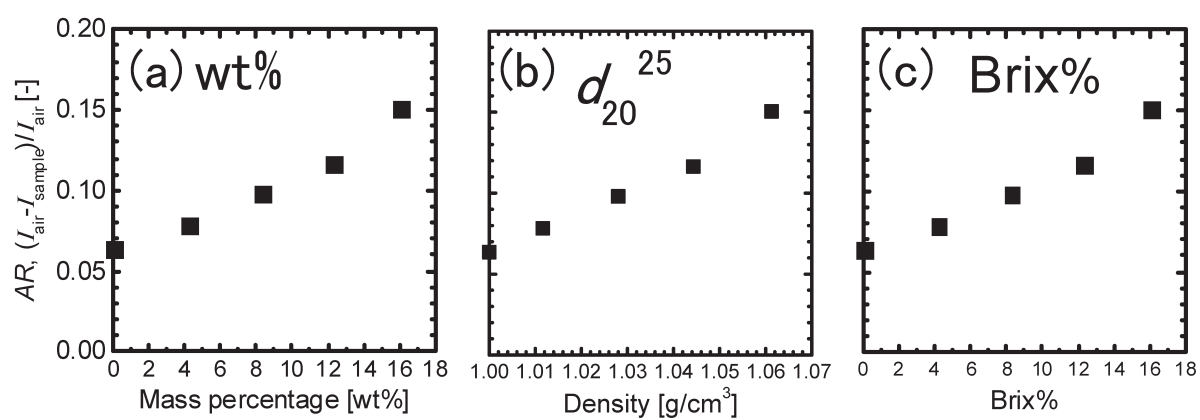

Figure 4 Relationship between $A R$ and mass percentage, density or Brix\% of sucrose aqueous solutions.
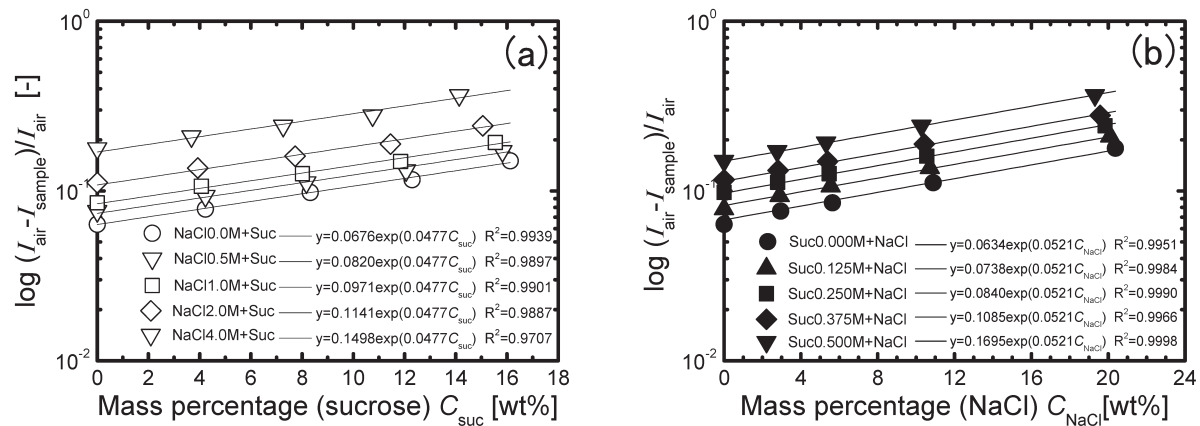

Figure 5 Relationship between $A R$ and sucrose or $\mathrm{NaCl}$ contents in mixtures of sugar-salt aqueous solutions.

This result suggested that the bent optical fiber could be used as the sensitive part of the sensor to measure the solute content in aqueous solutions. It is considered that the intensity of light through the bent optical fiber depends on the refractive index of the sample touching the sensitive part. In general, foods and the other biosystems have a complicated component structure such as a mixture of organic components and metal ions. Therefore, we checked the sensor performance using sucrose- $\mathrm{NaCl}$ aqueous solutions as the sample.

Figure 5 shows the measurement results of the $A R$ values when the sensitive part was soaked in the sucrose- $\mathrm{NaCl}$ aqueous solutions. The $A R$ value increased when the sucrose was added to the $\mathrm{NaCl}$ aqueous solutions (Figure 5(a)) and when the $\mathrm{NaCl}$ was added to the sucrose aqueous solutions (Figure 5(b)). The $A R$ value is indicated by a logarithmic scale in the figure and a good linearity was obtained. It seems that the $A R$ value exponentially increased with the content of the sucrose or $\mathrm{NaCl}$.

\subsection{Measurement of sugar content of culture broth during plant cell cultivation}

We used the developed sensor to measure the sugar content in the culture broth during plant cell cultivation which is one of the typical complicated composition samples as a mixture of sugar and salts. However, the plant cell cultivation broth only contains under $30 \mathrm{~g} / \mathrm{dm}^{3}$ sugar. Although it was confirmed that the constructed sensor and the experimental apparatus can measure the solute content in an aqueous solution, the content of the used samples for the fundamental performance test (the sugar-salt aqueous solutions) is higher than that in the actual foods and bio-systems. The detection of slight differences in the light intensity would be required for the bent optical fiber sensor. Therefore, we constructed a prototype sensor system using an LED (NSPL570DS, NICHIA Co., Tokushima, Japan), PD (OSD1-5T, Centronic Ltd., UK) and OP-amp (LM248N, STMicroelectronics. N. V., Geneva, Switzerland) for differential amplification (Figure 6). Although the constructed circuit is common differential amplifier, however, it was improved that the weak current from the photodiode is converted into the higher voltage. The main circuit was also constructed using a general purpose electric parts.

Figure 7 shows the correlation between the sugar contents of the culture broth measured by the FT-IR method and the sensor system using the experimental apparatus (gray triangles) or using the differential amplification circuit (black circles). The calculated results of the sugar contents using the experimental apparatus were not suitable for the culture broth of the plant cells. On the other hand, an excellent agreement between the FT-IR method and the sensor method using the OP-amp was observed. As the results, the time courses of the sugar content and the consumption rate were able to be calculated during the plant cell cultivation process (Figure 8). Good agreement of the calculated results was obtained between the FT-IR method (gray squares and dotted line) and the constructed sensor (black circles and line). 

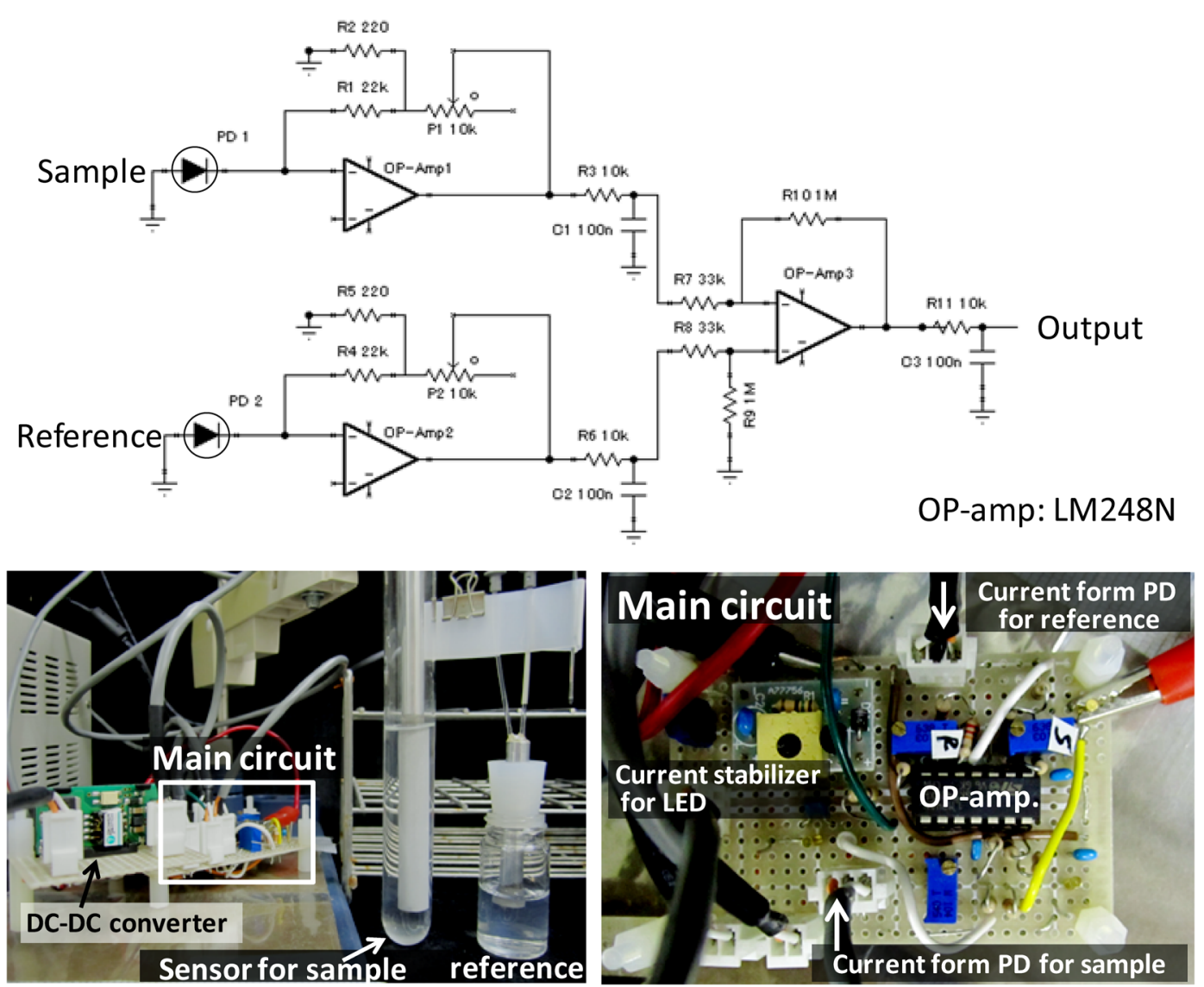

Figure 6 Prototype sensor system using LED, PD and differential amplifier (OP-amp.) with two bent optical fibers (for reference and sample).

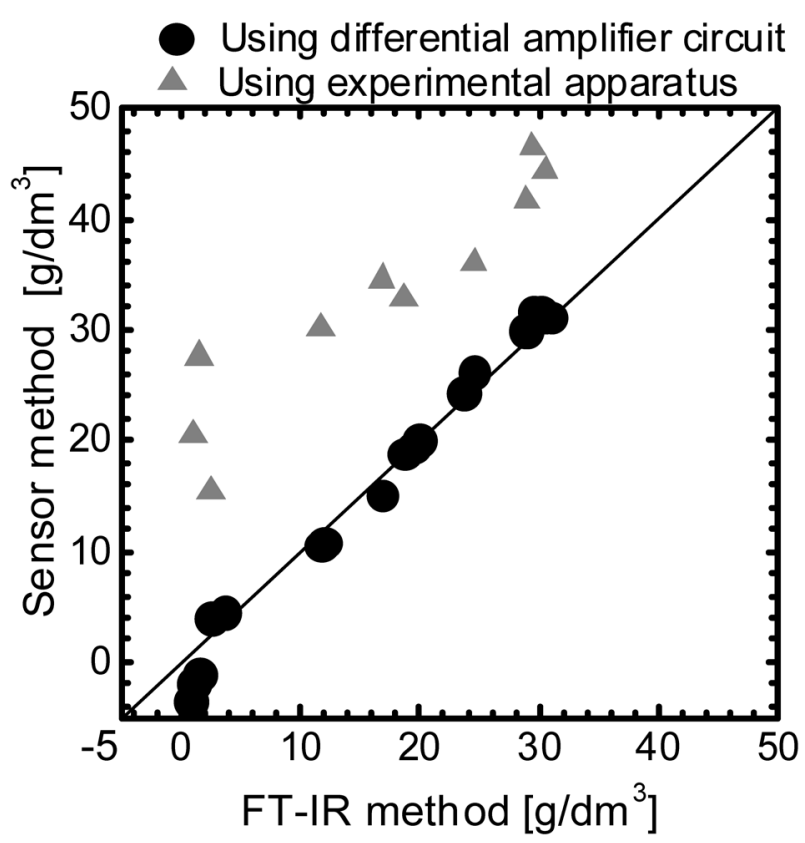

Figure 7 Correlation between sugar contents of culture media measured by FT-IR method and the sensor system using the experimental apparatus (gray triangles) or differential amplifier electric circuit constructed with LED and PD (black circles).

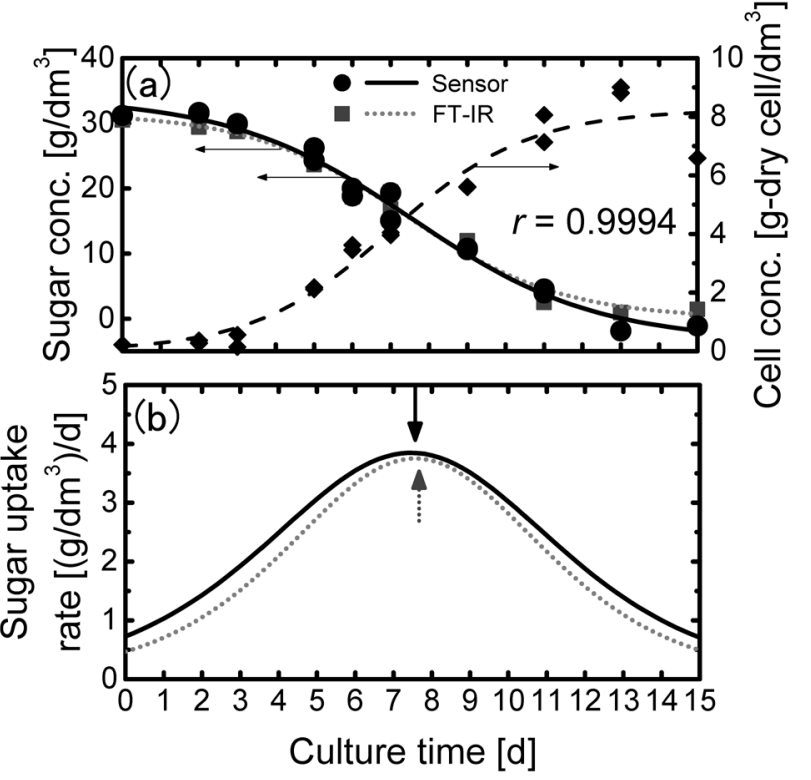

Figure 8 Time courses of the sugar content (a) and the uptake rate (b) measured and calculated by FT-IR method and the sensor system. 


\section{Discussion}

The bent optical fiber was used for measurement of the solute content in aqueous solutions (Figures 4 and 5). The differences in the light intensity when the reference material and the sample solution were contacted with the sensitive part was defined as the $A R$ value expressed by Eq. (2). The relationship between the calculated $\log (A R)$ values and the solute contents in the aqueous solution is a linear correlation. These results suggested that the $A R$ value obtained by the developed sensor is treated as the transmittance values using the Lambert-Beer law ${ }^{31}$. The law is normally described by the following equation

$$
A_{\lambda}=-\log \frac{I_{\lambda}}{I_{0, \lambda}}=-\log T_{\lambda}=\varepsilon_{\lambda} c L
$$

where $I_{0, \lambda}$ and $I_{\lambda}$ are the radiant intensity of light entering the sample and exiting the sample, respectively. The subscript $\lambda$ is the wavelength of light. The ratio, $I_{\lambda} I_{0 \lambda}$, is defined as the transmittance $\left(T_{\lambda}\right)$ and $A_{\lambda}$ is the absorbance of the sample. $\varepsilon_{\lambda}, c$, and $L$ are the absorption coefficient, which is specific to the kind of material the content of the target material in the sample, solute content in the sample, and the light pass length of the sample, respectively. The kind of material can be identified by the spectrum pattern because $\varepsilon_{\lambda}$ is different for every kind of material. At the same time, several materials in samples with a complicated composition can also be simultaneously measured because the absorbance $A_{\lambda}$ is proportional to the content $c$.

In Figure 5(a) and 5(b), the fitting results of the linear approximation (slope and intercept of the straight line) are also displayed. The slope for the sugar contents is slightly difference for the salt contents. The sucrose and sodium chloride contents of the aqueous solution used in this fundamental performance test is higher than the contents that are assumed to be in actual foods or biosystems, and the interaction between the sugar and salt will be more definitely observed in the optical sensing data $^{26)}$. The optical sensing data obtained by this bent optical fiber sensor will be used to recognize the kind of materials. In the case of the tested sample which is a transparent liquid, the obtained spectral information is an almost flat pattern because the light source for the visible radiation was used to construct the experimental apparatus. Figure 9 shows the observed spectra of two kinds of pigments $\left(0.025 \mathrm{~g} / \mathrm{dm}^{3}\right.$ rhodamine $\mathrm{B}$ and $0.025 \mathrm{~g} / \mathrm{dm}^{3}$ methylene blue) in aqueous solutions obtained by the experimental apparatus with the bent optical fiber sensor. Although the optical fiber of $3 \mathrm{~mm}$ in diameter was used to more strongly obtain the absorption the spectral information, which characterized the two kinds of pigment solutions, was obtained. These results suggested that the bent optical fiber could be used as an attenuate total reflection (ATR) immersion

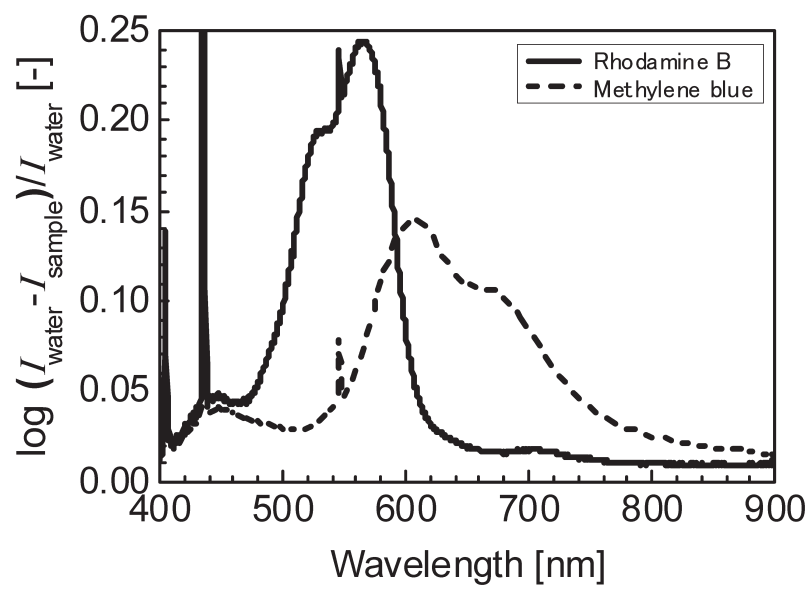

Figure 9 Spectra of two kinds of pigment aqueous solutions obtained from experimental apparatus with bent optical fiber.

type sensor probe. The processing of the sensor probe is very easy because the optical fiber was only bent. If a sensing material, such as a fluorescence enhancer for specific materials, polymer film, and a pigment that turns color depending on the $\mathrm{pH}$, is fixed to the bent optical fiber, various types of optical sensors, which were developed in the preliminary studies ${ }^{12,13)}$, are able to be developed. In addition, spectral information will be able to be simultaneously obtained. In general, the structure of the bio-related substrate may change depending on the environmental situations such as the $\mathrm{pH}$ and temperature ${ }^{32}$. The structure change in the bio-related substrate may influence the spectral information which is obtained by the optical sensing ${ }^{26,32)}$. Simultaneous measurement of the spectral information and the environmental situation will be tested in a future study.

The developed sensor and the experimental apparatus was used in an actual situation, such as a plant cell culture broth, and a slight difference in the light intensity between the reference (water) and the sample (culture broth) had to be detected because the sugar content in the culture broth were lower than that of the tested sample solutions. The prototype sensor using the OP-amp for differential amplification, two LEDs and two PDs was used to apply the bent optical fiber sensor in an actual situation. This prototype sensor can be constructed very cheaply because the sensor and the electronic parts are not expensive and the sensor system has a simple configuration. For the sensitive part of the sensor, the bent optical fiber, there is no processing to make the sensitive part except for the bending. The sensor system was able to be constructed only in a simple light detection circuit where the LED and PD were placed on both ends of the bent optical fiber.

Although the maximum values of the sugar content in the culture broth is under $30 \mathrm{~g} / \mathrm{dm}^{3}$, a good agreement between the calculated results using the sensor 
system and using the FT-IR method ${ }^{27,29)}$ was obtained (Figures 7 and 8). In both methods, the curve fitting for obtaining the time courses of the sugar content were possible using the logistic function, Eq. (1). In addition, the calculated results of the sugar uptake rate using the sensor system also corresponded to the calculated result using the FT-IR method ${ }^{30}$. However, a slight error was observed in the case of the measurement results using the developed sensor system. The reason for the error will be the influence of the small amount of salts and the trace elements (mineral sources) in the culture broth. The salt and the trace element contents of the culture broth could be estimated by measurement of the electric conductivity (EC) values, and the cell content in the culture broth is also able to be estimated by the EC values ${ }^{33)}$. The observed error may be corrected using the EC values of the culture broth. On the other hand, the sugar uptake rate is one of the important results for the bioprocess management of the cultivation. The monitoring of the sugar contents and the uptake rate are very important in order to analyze the sugar metabolism of the plant cells ${ }^{27,30)}$. In our future plans, we intend to control the growth and metabolism reaction of the plant cells by changing the carbon source (sugar) type in the middle of the cultivation stage without using the gene recombination technique. In other words, we consider this application as a simple sensing tool to regulate the growth and the metabolic reaction rate of the cells by controlling the environment during the plant cell cultivation. It is very important results that the time courses of the metabolic material contents could be expressed quantitatively for the bioprocess management. In general, the time courses of the content of the cells and the metabolic materials, such as cell growth, sugar uptake, and production of the metabolic products, during the fermentation process is able to be explained by the logistic function curves. In the results of the curve fitting using the Boltzmann equation (Eq. 1), good agreement was obtained between the calculated curve obtained by the sensor system and by the FTIR method because the correlation coefficient of the sugar contents and the sugar uptake rate curves were 0.9994 and 0.9976, respectively (Figure 8). As shown in Figure 8(b), slight error of the maximum sugar uptake rate and the time were observed, however, the relative error were about $2.5 \%$ for the maximum rate and $1.3 \%$ for the culture period of the peak time, respectively. These results suggested that the optical sensor system constructed by the bent optical fiber is applicable for the bioprocess monitoring of the sugar content.

The constructed simple optical sensor is one of the powerful tools for a simpler and easier bioprocess management. In addition, the structure of the proposed sensor system in this study is very simple, and a price reduction and disposable use of the sensor will be realized. The application to optical sensing under the condition of wide use such as at an agricultural production site and environmental measurement site using the characteristics of the optical fiber will be possible. The simple optical sensor is applicable as one of the disposable sensing tools for the sensor network in the IoT concept such as in a smart agriculture setting and smart factory.

\section{Concluding remarks}

A simple optical sensor using the bent optical fiber was developed for the optical sensing of the solute content in an aqueous solution without precise processing of the sensitive part. The attenuation ratio of the light intensity obtained by the constructed sensor was treated as the transmittance values of the optical sensing. In fact, the spectral information of two kinds of pigment aqueous solutions was able to be acquired using the bent optical fiber. However, a slight change in the attenuation ratio must be detected in a practical application such as the differences in the light intensity slightly changed between the reference materials (water) and the samples (culture broth). Therefore, we constructed a sensor system using an LED, PD and OP-amp for the differential amplification. As a result, measurement of the time courses of the sugar content during plant cell cultivation ranging from 0 to $30 \mathrm{~g} / \mathrm{dm}^{3}$ was possible. In addition, the sugar uptake rate of the plant cell during cultivation was able to be calculated and excellent agreement between the FT-IR method and the sensor method using an electric circuit was observed. These results suggested that the developed prototype sensor using a bent optical fiber is applicable as a simple and disposable optical sensor. The continuous measurement and the precious control of the sugar content in the fermentation process (plant cell cultivation) using developed sensor system will be carried out in the future.

\section{Acknowledgments}

This work was partly supported by a Grant-in-Aid for Challenging Exploratory Research 25630375 from the JSPS KAKENHI and by project to Adaptable and Seamless Technology Transfer Program through Target-driven R\&D (AS231Z02962B) from the Japan Science and Technology Agency (JST).

\section{References}

(1) Kameoka, T. and Hashimoto, A.: Effective application of ICT in Food and Agricultural sectorOptical sensing is mainly described-, IEICE Trans. Commun., E98-B-9, pp. 1741-1748 (2015).

(2) Li, W.-T., Majewsky, M., Abbt-Braun, G., Horn, H., Jin, J., Li, Q., Zhou, Q. and Li, A.-M.: Application 
of portable online LED UV fluorescence sensor to predict the degradation of dissolved organic matter and trace organic contaminants during ozonation, Water Res., 101, pp. 262-271 (2016).

(3) Murphy, K., Sullivan, T., Heery, B. and Regan, F.: Data analysis from a low-cost optical sensor for continuous marine monitoring, Sens. Actuators B Chem., 214, pp. 211-217 (2015).

(4) Suehara, K. and Yano, T.: Bioprocess monitoring using near-infrared spectroscopy, Adv. Biochem. Engin. Biotechnol., 90, pp. 173-198 (2004).

(5) Shimazaki, T. and Hara, S.: Design of PPG-based heart rate sensor enabling motion airtiface cancellation, SASIMI 2015 proceedings, pp. 283-286 (2015).

(6) Selvam, A. P., Muthukumar, S., Kamakoti, V. and Prasad, S.: A wearable biochemical sensor for monitoring alcohol consumption lifestyle through ethyl glucuronide $(\mathrm{EtG})$ detection in human sweat, Sci. Rep., 6-1, p. 23111 (2016). DOI: 10.1038/srep23111

(7) Ylijoki, O. and Porras, J.: Conceptualizing big data: Analysis of case studies, Intell. Sys. Acc. Fin. Mgmt. (2016). DOI: 10.1002/isaf.1393

(8) Bronson, K. and Knezevic, I.: Big data in food and agriculture, Big Data \& Society (2016). DOI: $10.1177 / 2053951716648174$

(9) Leung, C. K. Y., Wan, K. T., Inaudi, D., Bao, X., Habel, W., Zhou, Z., Ou, J., Ghandehari, M., Wu, H. C. and Imai, M.: Review: Optical fiber sensors for civil engineering applications, Mater. Struct., 48-4, pp. 871-906 (2015).

(10) Arifin, A., Hatta, A. M., Munitini, M. S. and Rubiyanto, A.: Bent of plastic optical fiber with structural imperfections for displacement sensor, Indian J. Pure Appl. Phy., 52, pp. 520-524 (2014).

(11) García, J. A., Monzón-Hernández, D., Manríquez, J. and Bustos, E.: One step method to attach gold nanoparticles onto the surface of an optical fiber used for refractive index sensing, Opt. Mater., 51, pp. 208-212 (2016).

(12) Hernández-Romano, I., Miguel, A. C.-G., MorenoHernández, C., Monzón-Hernández, D., LópezFigueroa, E. O., Paredes-Gallardo, O. E., TorresCisneros, M. and Villatoro, J.: Optical fiber temperature sensor based on a microcavity with polymer overlay, Opt. Express, 24-5, pp. 5654-5661 (2016). DOI: 10.1364/OE.24.005654

(13) Woyessa, G., Nielsen, K., Stefani, A., Markos, C. and Bang, O.: Temperature insensitive hysteresis free highly sensitive polymer optical fiber Bragg grating humidity sensor, Opt. Express, 24-2, pp. 1206-1213 (2016). DOI: 10.1364/OE.24.001206

(14) Suehara, K., Tsunematsu, T., Tasaka, T., Kohda, J., Nakano, Y., Fujii, E. and Yano, T.: Development of an air-oil and oil-water interface detector using op- tical fiber and its application for measurement of oil layer thickness of industrial kitchen wastewater in a grease trap, J. Chem. Eng. Japan, 39-6, pp. 670-677 (2006).

(15) Okuda, S., Okajima, K. and Arce-lopera, C.: Visual palatability for food dishes in color appearance, glossiness and convexo-concave perception depending on light source, J. Light Vis. Env., 39-0, pp. 8-14 (2015).

(16) Yasuda, T., Hayakashi, T., Naoki, S., Maehara, A., Hiramatsu, K., Kido, S. and Hayashi, Y.: Light sources and lighting circuits, J. Light Vis. Env., 363, pp. 106-120 (2012).

(17) Jishi, T., Fujiwara, K., Nishino, K. and Yano, A.: Pulsed light at lower duty ratios with lower frequencies is less advantageous that continuous light for $\mathrm{CO}_{2}$ uptake in Cos Lettuce, J. Light Vis. Env., 36-3, pp. 88-93 (2012).

(18) Sansalvador, I. M. P. V., Fay, C. D., Cleary, J., Nightingale, A. M., Mowlem, M. C. and Diamond, D.: Autonomous reagent-based microfluidic $\mathrm{pH}$ sensor platform, Sens. Actuators B Chem., 225, pp. 369-376 (2016).

(19) Li, W. T., Jin, J., Li, Q., Wu, C. F., Lu, H., Zhou, Q. and Li, A. M.: Developing LED UV fluorescence sensors for online monitoring DOM and predicting DBPs formation potential during water treatment, Water Res., 93, pp. 1-9 (2016).

(20) Neto, S. Y., Luz, R. C. S. and Damos, F. S.: Visible LED light photoelectrochemical sensor for detection of L-Dopa based on oxygen reduction on $\mathrm{TiO}_{2}$ sensitized with iron phthalocyanibe, Electrochem. Commun., 62, pp. 1-4 (2016).

(21) Rocchitta, G., Spanu, A., Babudieri, S., Latte, G., Madeddu, G., Galleri, G., Nuvoli, S., Bagella, P., Demartis, I. M., Fiore, V., Manetti, R. and Serra, P. A.: Enzyme biosensors for biomedical applications: Strategies for safeguarding analytical performances in biological fluids, Sensors 2016, Vol. 16, pp. 780-801 (2016). DOI: 10.3390/s16060780

(22) Li, J., Koinkar, P., Fuchiwaki, Y. and Yasuzawa, M.: A fine pointed glucose oxidase immobilized electrode for low-invasive amperometric glucose monitoring, Biosens. Bioelectron., 86, pp. 90-94 (2016).

(23) Nishitani, S., Kajisa, T. and Sakata, T.: Development of molecularly imprinted polymer-based field effect transistor for sugar chain sensing, Jpn. J. Appl. Phys., 56 (2017). DOI: 10.7567/JJAP.56.04CM02

(24) Anzai, J:: Recent progress in electrochemical biosensors based on phenylboronic acid and derivatives, Mater. Sci. Eng. C, 67, pp. 737-746 (2016). DOI: 10.1016/j.msec.2016.05.079

(25) Çiftçi, H., Alver, E., Çelik, F., Metin, A. Ü. and Tamer, U.: Non enzymatic sensing of glucose using a glassy carbon electrode modified with gold 
nanoparticles coated with polyethyleneimine and 3-aminophenylboronic acid, Microchem. Acta, 1834, pp. 1479-1486 (2016).

(26) Kanho, M., Suehara, K., Kameoka, T. and Hashimoto, A.: Mid-infrared spectroscopic analysis of saccharides in aqueous solutions with sodium chloride, Biosci. Biotechnol. Biochem., 81-4, pp. 735-742 (2017).

(27) Suehara, K., Kameoka, T. and Hashimoto, A.: Sugar uptake analysis of suspension Arabidopsis, tobacco, and rice cells in various media using an FT-IR/ATR method, Bioprocess Biosyst. Eng., 358, pp. 1259-1268 (2012).

(28) Murashige, T. and Skoog, F.: A revised medium for rapid growth and bioassay with tabacco tissue culture, Physiol. Plant., 15-3, pp. 473-497 (1962).

(29) Hashimoto, A. and Kameoka, T.: Mid-infrared spectroscopic determination of sugar contents in plant- cell culture media using an ATR method, Appl. Spectrosc., 54-7, pp. 1005-1011 (2000).

(30) Hashimoto, A., Nakanishi, K., Motonaga, Y. and Kameoka, T.: Sugar metabolic analysis of suspensions of plant cells using an FT-IR/ATR method, Biotechnol. Prog., 17-3, pp. 560-564 (2001).

(31) Beer, A.: Bestimmung der absorption des rothen lichts in farbigen flussigkeiten, Ann. Phys., 162-5, pp. 78-88 (1852).

(32) Hashimoto, A., Sugimoto, Y., Suehara, K. and Kameoka, T.: Influences of $\mathrm{pH}$ and temperature on infrared spectroscopic features of brewed coffee, Procedia Food Sci., 1, pp. 1132-1138 (2012).

(33) Suehara, K., Takao, S., Nakamura, K., Uozumi, N. and Kobayashi, T.: Optimal expression of GUS gene from methyl jasmonate-inducible promoter in high density culture of transformed tobacco cell line BY-2, J. Ferment. Bioeng., 82-1, pp. 51-55 (1996). 